\title{
Inhibition of Aromatase Induces Partial Sex Change in a Cichlid Fish: Distinct Functions for Sex Steroids in Brains and Gonads
}

\author{
Carolin Göppert $^{\mathrm{a}}$ Rayna M. Harris ${ }^{\mathrm{b}, \mathrm{c}}$ Anya Theis ${ }^{\mathrm{a}}$ Anna Boila ${ }^{\mathrm{a}}$ Simon Hohla \\ Attila Rüegg $^{\text {a Hans A. Hofmann }}{ }^{\text {b-d }}$ Walter Salzburger ${ }^{\text {a }}$ Astrid Böhne $^{a}$ \\ a Zoological Institute, University of Basel, Basel, Switzerland; ${ }^{b}$ Department of Integrative Biology, ${ }^{\mathrm{C}}$ Institute for Cell \\ and Molecular Biology, and ${ }^{\mathrm{d} I n s t i t u t e}$ for Neuroscience, The University of Texas at Austin, Austin, Tex., USA
}

\section{Key Words}

Aromatase · Astatotilapia burtoni - Cichlid fish · Sex change . Sex differentiation $\cdot$ Sex steroids

\begin{abstract}
Sex steroids are major drivers of sexual development and also responsible for the maintenance of the established gender. Especially fishes exhibit great plasticity and less conservation in sex determination and sexual development compared to other vertebrate groups. In addition, fishes have a constant sex steroid production throughout their entire lifespan, which makes them particularly susceptible to interferences with the endogenous sex steroid system. This susceptibility has recently been used to show that inhibition of the key enzyme of estrogen synthesis, aromatase Cyp19a1, can induce functional sex reversal even in adult fish. Here, we investigated the impact of the aromatase inhibitor (Al) fadrozole in adult females of the East African cichlid fish Astatotilapia burtoni. Using gene expression, phenotypic measurements, behavioral experiments, and hormone measurements, we assessed if females treated with fadrozole develop a male-like phenotype. We found that Al treatment has a different effect on gene expression in the gonad compared to the brain, the 2 tissues mostly implicated in sexual develop-
\end{abstract}

ment. In contrast to observations in other gonochoristic species, A. burtoni ovaries cannot be transformed into functional testis by Al. However, rapid changes towards a male-like phenotype can be induced with $\mathrm{Al}$ in coloration, hormone levels, and behavior.

(c) 2016 S. Karger AG, Base

Most vertebrates are gonochoristic, that is the initially bipotential gonad develops permanently into either testis or ovary after a certain point in time. In agreement with this, the vast majority of the $\sim 30.000$ described teleost fish species [Nelson, 2006] are gonochorists [Avise and Mank, 2009]. While the mechanisms underlying sex determination and sexual differentiation are generally highly conserved in tetrapods, they are remarkably plastic in fishes and can be subject to frequent evolutionary turnovers [reviewed in Heule et al., 2014a].

Sex differentiation is mediated by the interplay of brain and gonad which is regulated through the production of gonadotropins in the pituitary and sex steroid hormones [Nagahama, 1994]. In fishes, sex steroid hormones

\section{A.B. and S.H. contributed equally to this work.}

\section{KARGER}

E-Mail karger@karger.com

www.karger.com/sxd
(C) 2016 S. Karger AG, Basel

1661-5425/16/0102-0097\$39.50/0 
are synthesized mainly in the gonad [Miller, 1988; Payne and Hales, 2004] but can also be produced in the brain [Schmidt et al., 2008]. Two major types of androgens are present in fishes, testosterone (T) and 11-ketotestosterone (11-KT), the latter being important for testicular development as well as the development of secondary sexual characteristics [Borg, 1994; Baroiller et al., 1999]. Estradiol is responsible for inducing and maintaining ovarian development [Baroiller et al., 1999] and is produced via the aromatization of $\mathrm{T}$ by the aromatase enzyme Cyp19a1. Fishes have 2 gene copies for this enzyme, cyp19a1a and cyp19a1b [Piferrer and Blázquez, 2005], which emerged from the teleost specific genome duplication [Böhne et al., 2013]. Cyp19a1a is usually highly expressed in the gonad whereas cyp $19 a 1 b$ is higher expressed in the brain [Böhne et al., 2013]. Overall, aromatase activity in the brain of fishes is reported to be 100 1,000 times higher than in other vertebrate taxa [Forlano et al., 2006]. Cyp19a1b is probably involved in a negative feedback control loop in the brain [Devlin and Nagahama, 2002], which allows steroid hormones to downregulate their own production via kisspeptin and the hypothalamic-pituitary-gonadal axis [Hofmann, 2006].

This plasticity in sexual development also means that aberrant sex reversal can be easily induced in fishes due to e.g. pollution [for reviews, see Bhandari et al., 2015; Senthilkumaran, 2015]. This is mainly due to interference with endogenous sex steroid hormones and can induce full and functional sex reversal when applied before the critical time window of (gonadal) sex differentiation. This phenomenon is routinely exploited in aquacultural mono-sex farming of fish species with a pronounced sexual dimorphism in weight and size or growth rate [Green and Teichert-Coddington, 2000].

Recent studies of induced sex change support the existence of an even greater plasticity of sexual development and identity in fishes than anticipated. A complete functional sex reversal can be induced by treatment with aromatase inhibitors (AI) even long after the original sex differentiation time window in adult females of three gonochoristic teleosts, medaka (Oryzias latipes), Nile tilapia (Oreochromis niloticus) [Paul-Prasanth et al., 2013; Sun et al., 2014], and zebrafish (Danio rerio) [Takatsu et al., 2013].

Here, we investigated if sex reversal can be induced in Burton's Mouthbrooder (Astatotilapia burtoni), a member of the most species rich lineage of cichlids, the Haplochromini, and endemic to East African Lake Tanganyika and its affluent rivers [Salzburger et al., 2005; Theis et al., 2014]. It is a well-studied model system in neuroen- docrinology [Huffman et al., 2013; Maruska, 2014], ecology [Theis et al., 2014] and behavioral research [Theis et al., 2012], in evo-devo [Santos et al., 2014], and in genetics and genomics [Salzburger et al., 2008; Böhne et al., 2013] with the benefit of a fully sequenced genome [Brawand et al., 2014]. A. burtoni is a gonochoristic maternal mouthbrooder species with pronounced sexual dimorphism. Males are larger, territorial, brightly colored, and have egg-spots (conspicuous orange-yellow markings) on the anal fin [Heule and Salzburger, 2011; Santos et al., 2014] and eye bars across their face [Renn et al., 2012]. Females are smaller, have cryptic coloration, and less pronounced yellow egg-spots. Using hormonal sex reversal experiments in juvenile fish with subsequent crossings, we could previously show that $A$. burtoni likely possesses an XX/XY sex chromosome system [Heule et al., 2014b].

Furthermore, this system has the advantage of a wellinvestigated sex steroid hormone system. Androgens modulate reproductive, aggressive, and dominant behavior in male A. burtoni [O'Connell and Hofmann, 2012; O'Connell et al., 2013], and estrogens increase aggressive behavior [Huffman et al., 2013]. Males have higher circulating levels of androgens and even $17 \beta$-estradiol (E2) than females [O'Connell et al., 2013] and levels of T and 11-KT show a positive correlation, i.e. 11-KT levels are lower than those of T [Kidd et al., 2010, 2013]. Furthermore, fadrozole has been shown to successfully inhibit aromatase in males of this species, leading to an increase in $\mathrm{T}$ levels and a reduction in E2 levels shortly after administration of this AI [Huffman et al., 2013].

Interestingly, the expression pattern of aromatase is different in A. burtoni compared to all other teleosts studied so far. Cyp19a1a is overexpressed in the ovary, while cyp19a1b is expressed in the brain (the classic expression pattern in teleosts). Additionally, cyp19a1b is overexpressed in the testis [Böhne et al., 2013]. The coding sequence of cyp 19a1b is conserved in cichlids, indicating a preserved function in aromatization of androgens, whereas the function of cyp 19a1a remains to be verified, since its coding sequence shows substantial changes in the haplochromine lineage [Böhne et al., 2013].

To determine whether fadrozole induces a sex change in adult females of $A$. burtoni, we used color and body measurements, behavioral experiments, as well as hormone and gene expression levels. We compared treated fish to untreated males and females to investigate potential changes in sex-specific characteristics. 


\section{Materials and Methods}

\section{Animals and Fadrozole Exposure}

All fish used in this study were sexually mature and obtained from a laboratory strain of $A$. burtoni. Fish were maintained at $25^{\circ} \mathrm{C}$ with a $12: 12 \mathrm{~h}$ dark-light cycle with 10 -min dusk and dawn periods. Tanks were provided with gravel and terracotta flowerpots, which served as natural shelters. To allow an individual identification, all fish were marked using a subdermal injection of a visible implant elastomer tag (Northwest Marine Technology).

Fish were divided into the following 4 experimental groups: female control group $(n=17)$, male control group $(n=10$ due to larger size and increased aggression levels), and female fadrozole AI group with 2 replicates in separate tanks (AI1, $n=17$; AI2, $n=$ 17). All fish were reared under the same conditions except for provided food. All groups were fed 3 times a day with a measured diet of flake food (TetraMin). The amount was adjusted weekly based on the overall weight of each group $(0.025 \mathrm{~g}$ of food/g wet body weight). After 70 days, the food amount was reduced by $50 \%$ due to saturation of food intake. Food for the AI groups was soaked in $100 \%$ ethanol containing fadrozole $(200 \mu \mathrm{g} / \mathrm{g}$ flakes, Sigma Aldrich), and for the control group it was soaked in $100 \%$ ethanol only. Food was dried at $37^{\circ} \mathrm{C}$ and stored at $4^{\circ} \mathrm{C}$. After 90 days of treatment, the remaining fish of the 2 AI replicates were combined into 1 tank.

Throughout the fadrozole treatment time, fish were visually inspected on a daily basis and time points of performed experiments adapted according to observed phenotypical and behavioral changes. For more in-depth analysis, we decided to track changes (sex steroid production and coloration) on a subset of the same individual fish.

\section{Development of Egg-Spots and Coloration}

The whole body and the anal fin were photographed weekly for 5 control females, and 5 fish of AI1 and AI2 under standardized measurement conditions using a Nikon D5000 digital camera. Five males of the control group were photographed before the experiment. All individuals were photographed after 90 days.

Egg-spot number and coloration were quantified and assigned to categories (see online suppl. fig. 1 for examples of the used eggspot coloration categories; for all online suppl. material, see www. karger.com/doi/10.1159/000445463). A linear mixed effect model (CRAN package lme4 version 1.1-6) [Bates et al., 2015] with repeated measures was used to examine treatment effects over time on egg-spot number and coloration, which were set as the dependent variable. Treatment groups and day of treatment and their interaction were included as fixed effects, and individual identity was included as random effect. Data was analyzed following the methods described for the hormone measurements.

\section{Morphometrics of Sex-Specific Shape Differences}

To quantify changes in body shape over time, geometric morphometric analyses were conducted on photographs from day 0 and day 90 of control and treated individuals (see online suppl. table 1 for sample sizes). A file in .tps format was created with tpsUtil (v.1.60) [Rohlf, 2015a]. Coordinates of 7 homologous landmarks representing overall body shape and 5 homologous landmarks plus 4 landmarks defining the eye were digitized using tpsDig2 (v.2.18) [Rohlf, 2015b] (for landmark positions, see online suppl. fig. 2). A constructed central eye landmark was subsequently calculated as mean value of the 4 eye landmarks. Final coordinates ( 7 body coordinates, 6 head coordinates) were imported into MorphoJ (v.1.06d) [Klingenberg, 2011], and landmark configurations were superimposed by a generalized procrustes analysis to remove size variation and rotational as well as translational differences.

A principal component analysis (PCA) was then used to evaluate variation in body and head shape between specimens. Scatterplots were produced showing group differences in body and head shape before the experiment (day 0) and after 90 days. Landmarkbased measurements were used to calculate head length, standard length, and body height.

We further calculated relative body height (height/standard length) and relative head length (head length/height). Mean size differences in relative body height and relative head length were tested using a 1-way analysis of variance (ANOVA) and a Tukey HSD post hoc test.

\section{Hormone Assays}

Waterborne hormone levels were measured at 4 time points (before treatment administration, after 14, 30, and 90 days) from 5 fish of the female control group and 5 individuals each for the treatment groups AI1 and AI2. Waterborne hormone levels for 5 males at 0 days were assessed as baseline levels. Waterborne T, E2, and 11-KT levels were quantified following the method validated for $A$. burtoni by Kidd et al. [2010] using enzyme immune assay kits from Cayman Chemical Company (T EIA kit 582701, 11-KT EIA kit 582751, E2 EIA kit 582251) following the manufacturer's instructions. Briefly, water samples were filtered and immediately processed using C18 solid-phase extraction columns (Waters Corporation) attached to a vacuum manifold and stored afterwards at $-20^{\circ} \mathrm{C}$. Samples were eluted with ethanol, dried under nitrogen gas, and suspended in assay buffer. E2 and T samples were diluted 1:16 in assay buffer and 11-KT samples were diluted 1:4. Absorbance was measured at $405 \mathrm{~nm}$ using a micro plate reader (Spectramax M2e Plate Reader). All samples and standards were assayed in duplicate, and 2 plates each were run for T, E, and 11-KT due to sample size.

The intra-assay coefficients of variation were below the general tolerance level of $20 \%$ [Reed et al., 2002] with 3.1 and $2.7 \%$ for $\mathrm{T}, 3.6$ and $2.7 \%$ for E, and 8.56 and $5.2 \%$ for $11-\mathrm{KT}$. The inter-assay coefficients of variation for $\mathrm{T}, \mathrm{E}$, and $11-\mathrm{KT}$ were $4.4,6.6$, and $12.5 \%$, respectively. Values with a $\% \mathrm{CV}>20$ were excluded. All statistical analyses were conducted in R (v.3.0.2) [R Core Team, 2014]. For all experiments, variables were visually inspected by examining QQ plots and tested for normality using a Shapiro-Wilk test. For subsequent statistical analyses, hormone measurements were log-transformed, resulting in normalized residuals. A linear mixed effect model (R CRAN package lme4 version 1.1-6) with repeated measures was used to examine treatment effects over time on hormone concentration. Hormone levels (T, 11-KT, E2) were set as the dependent variable, treatment groups and days of treatment as factor, and their interaction were included as fixed effects. To account for repeated measures, individual identity was included as random effect. The best fitting model was selected based on Akaike information criterion and a $\chi^{2}$ test (ANOVA). To compare time points between groups, a comparison matrix was used ( $R$ CRAN package multcomp version 1.4-0) [Hothorn et al., 2008]. Subsets were used to examine time points within a group. Plots were generated using the R package ggplot2 (version 1.0.1) [Wickham, 2009]. 
Gonad Histology

Gonad histology was assessed in 3 individuals per treatment group after 168 days. Gonads were dissected and fixed overnight in $4 \%$ paraformaldehyde at $4^{\circ} \mathrm{C}$. Samples were subsequently washed in $70 \%$ ethanol and kept in ethanol for storage at room temperature. Histological analyses were done by Histalim (Montpellier) on 3-5 $\mu \mathrm{m}$ gonad sections using a hematoxylin-eosin staining. Gonad type analyses were based on structural aspects, mainly the presence or absence of ovarian follicles and oocytes. Slides were digitalized (Nanozoomer 2.0HT, Hamamatsu) and assessed using the NanoZoomer Digital Software (NDP.view 2). The gonado-somatic index (GSI) was calculated as GSI = (gonad weight/body wet weight $) \times 100$.

\section{RNA Extraction, cDNA Synthesis, and $q R T-P C R$}

Expression levels of genes with described sexual dimorphic expression patterns as well as genes involved in the sex steroid pathway were measured in brain and gonad tissue. RNA extraction and expression analysis followed the method described in Böhne et al. [2013]. Briefly, gills, brain tissue, and gonads of each sex and group (females, $\mathrm{n}=5$; males, $\mathrm{n}=5$; AI 168 days, $\mathrm{n}=3$, AI 310 days, $\mathrm{n}=$ 6) were dissected and stored at $-80^{\circ} \mathrm{C}$ in RNAlater (Life Technologies) until extraction.

Samples were incubated overnight at $4^{\circ} \mathrm{C}$ in Trizol (TRI Reagent, Sigma Aldrich), and RNA was subsequently extracted following the Trizol protocol and stored at $-80^{\circ} \mathrm{C}$. RNA quality and quantity were assessed by measuring spectrophotometric absorbance (NanoDrop1000, Thermo Scientific) and using a fluorometer (Qubit RNA BR Assay Kit, Life Technologies). Prior to reverse transcription, RNA was treated with DNA-free Kit (Life Technologies) following the manufacturer's instructions to remove potential DNA contaminations. DNase-treated RNA $(1 \mu \mathrm{g})$ was reversetranscribed into cDNA using the High Capacity RNA to cDNA Kit (Applied Biosystems).

All qRT-PCR experiments were run on a StepOnePlus RealTime system (Life Technologies) with a final cDNA concentration of $0.5 \mathrm{ng} / \mu \mathrm{l}$ and $200 \mathrm{nM}$ primer with SYBR Green master dye (Roche) in a total volume of $20 \mu \mathrm{l}$ using the following cycling conditions: $95^{\circ} \mathrm{C}$ for $10 \mathrm{~min}, 40$ cycles of $95^{\circ} \mathrm{C}$ for $15 \mathrm{~s}$ and $58^{\circ} \mathrm{C}$ for $60 \mathrm{~s}$. A melt curve step after cycling was included for all amplifications to insure specificity of amplification.

Primer sequences for $a r a$ and $a r b$ were taken from Harbott et al. [2007] and validated as previously described [Böhne et al., 2013]. Primers and their efficiencies for cyp19a1A, cyp19a1B, cyp11b2, foxl2A, foxl2B, sf-1, dmrt1, and rpl7 were taken from Böhne et al. [2013]. For each gene, all samples were run with 3 technical replicates. For data analysis, the lowest cycle threshold (Ct) for expression was set at 37 cycles. Expression of the target gene was calculated relative to the control gene $r p l 7$ by using the equation for mean normalized gene expression: $\mathrm{MNE}=\mathrm{E}_{\mathrm{rpl}}{ }_{\text {Ctrpl7 }} / \mathrm{E}_{\text {Target }}{ }_{\text {CtTarget }}$ [Simon, 2003]. To compare gene expression data between control males, control females, and AI females, a non-parametric KruskalWallis test on ranks with a Dunn's post hoc test was performed ( $R$ CRAN package dunn.test version 1.2.3), following the method described in Maruska and Fernald [2010]. A Benjamini-Hochberg false discovery rate was used to correct for multiple comparisons.

\section{Behavior}

A 2-way choice experiment was used to test if fadrozole induced a shift from female towards male behavior in AI females after 250 days of treatment. Fadrozole treatment was paused for the duration of the behavioral trial to avoid cross contamination of experimental groups. A 3-tank set up with a larger central $(60 \times$ $30 \times 30 \mathrm{~cm})$ and 2 smaller outer tanks $(40 \times 25 \times 25 \mathrm{~cm})$ was used to allow choices based solely on visual cues (online suppl. fig. 3 ) [Theis et al., 2012].

Fish were weighted, standard and total length were measured, and fish pairs were assigned based on similar size. A control male and a control female stimulus fish were simultaneously presented in the outer tanks to a control female, control male, or AI female focal fish placed in the central tank. Sex of the stimulus fishes was assigned randomly to the 2 outer tanks to control for a possible side bias. Fish were allowed to acclimate for at least $19 \mathrm{~h}$ prior to filming. All tanks were provided with shelter, and fish were visually isolated until the filming was performed. Ten minutes of each video (Sony Handycam HDRXR550VE) were analyzed (iMovie, v. 9.0.8), starting after the focal fish visited both choice zones and returned to the neutral zone. Interaction time and time spent in choice zones were measured.

Attempts of interaction, number of visits in choice zone, presence/absence of an eye bar within choice zones, and display behavior (lateral and frontal display of spread fins) of the focal fish were counted. A Friedman test was used to correct for the multiple use of stimulus pairs. A Wilcoxon signed-rank test with Bonferroni correction was performed as post hoc analysis.

\section{Results}

\section{Fadrozole Rapidly Induces Male-Typical Coloration in}

\section{A. burtoni}

Early on in the experiment (after 14 days), we observed the appearance of male-typical coloration in AI females. They showed an increase in egg-spot number and size (fig. 1A, C, 14 days), and we detected a significant interaction between $\mathrm{AI}$ treatment and day of treatment for egg-spot number [lme: $\mathrm{F}(9,14)=30.79, \mathrm{p}<$ 0.001 ] as well as egg-spot coloration [lme: $\mathrm{F}(9,14)=$ 73.53, $\mathrm{p}<0.001]$.

Egg-spot number of AI females started to differ significantly from control females between 14 and 28 days $(\mathrm{z}=2.37, \mathrm{p}=0.036)$, and egg-spot coloration differed already between 0 and 14 days $(\mathrm{z}=2.44, \mathrm{p}=0.014)$. After 28 days, egg-spots showed an orange coloration and increased further in size in AI females. After 56 days, AI females developed additional egg-spots, which were orange and surrounded by an outer blue circle. No further changes in egg-spots were detected after 90 days. After 90 days, AI females displayed male territorial coloration, with most individuals displaying an eye bar, male-like egg-spots, and male body coloration. In contrast to this, control females did not change in egg-spot number or coloration (fig. 1B, C). 


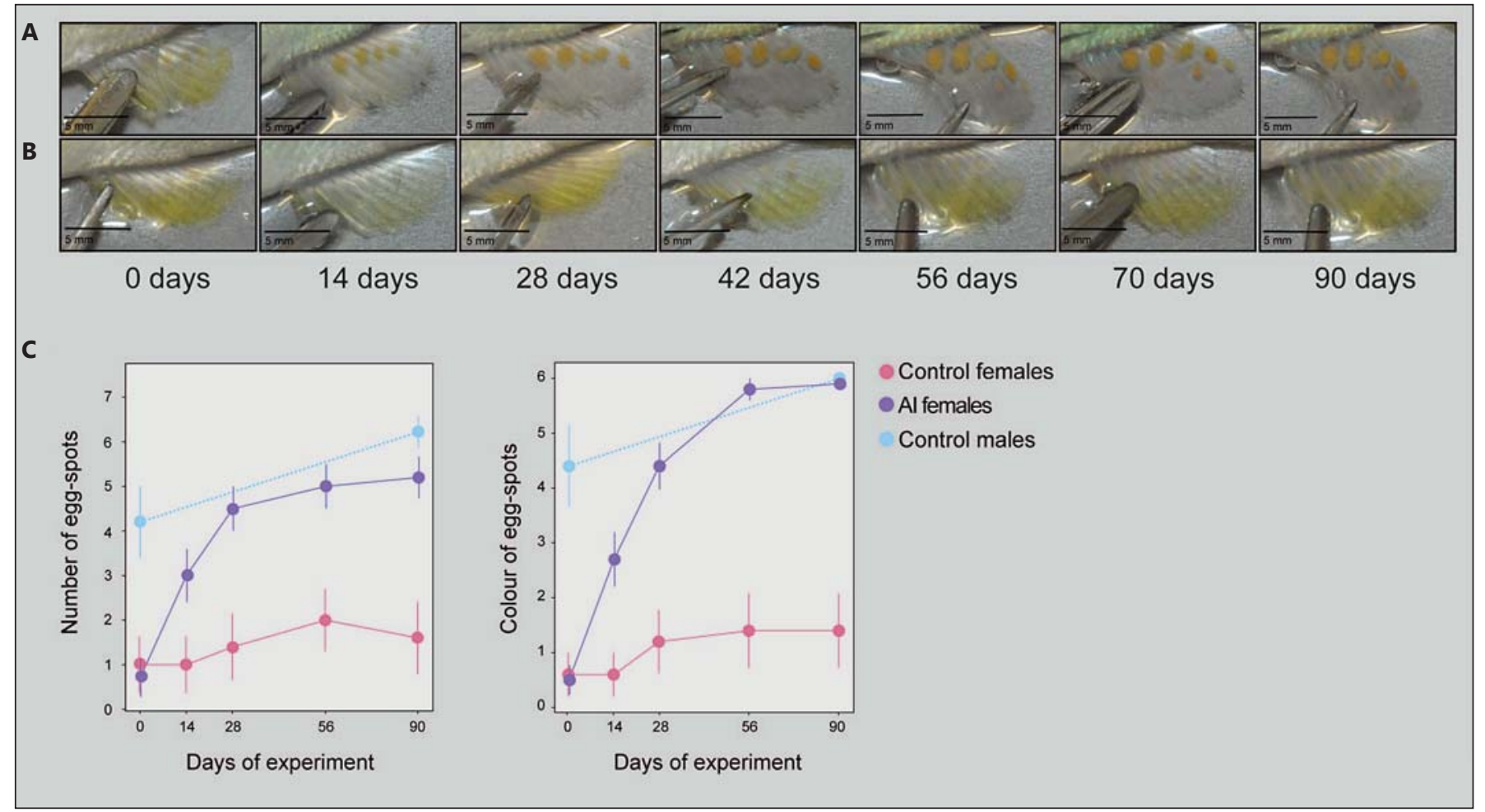

Fig. 1. Development of egg-spots in AI females. Picture series showing the anal fin of an AI female (A) and a control female (B) throughout 90 days. C Development of egg-spot number and color throughout the first 90 days of the experiment (mean \pm SEM).

\section{Morphometrics of Sex-Specific Shape Differences}

Only 2 of the 5 control males showed territorial coloration before the experiment (day 0), likely due to the mono-sex set-up of the tanks. None of the control or of the AI females showed territorial coloration at day 0 (fig. 2A, 3A). Territorial coloration was defined as the presence of at least 3 of the following traits: eye bar, orange egg-spots, body coloration, and blue lips. After 90 days, all males and $88 \%$ of AI females showed clear territorial coloration, whereas none of the females showed this trait combination.

To assess if the overall body shape was affected by AI and if body shape could be driven towards a more malelike body, we used geometric morphometrics on images of control and AI females at day 0 (same individuals for which hormone production was measured, see below), images of all female fish used in the experiment after 90 days, and images of the male control group before and after 90 days (online suppl. table 1). The 2 AI replicate groups did not vary in their distribution of individuals in morphospace nor showed significant differences [relative head length, ANOVA: $F(3,16)=0.7256, \mathrm{p}=0.551$; relative body length, ANOVA: $\mathrm{F}(3,16)=0.4771, \mathrm{p}=0.7227]$ and were hence pooled in this analysis. In a PCA of mean body shape, PC 1 (principal component) and PC 2 combined covered $59.9 \%$ of the total variation in the dataset. Group differences in body shape along PC 1 (fig. 2A) were mainly based on body depth, dorsal fin length, and - to some extent - mouth position. All groups showed substantial overlap at day 0 . After 90 days, all groups exhibited a deeper body shape with a shorter dorsal fin (higher PC 1 values).

At 90 days, differences between control groups were largely confined to PC 2, with females being more slender and having a more elongated head as males, which in addition showed a prolongation of the dorsal and anal fin. Interestingly, AI females showed an intermediate body shape, overlapping with both males and females on PC 1 and PC 2 after 90 days.

Comparing relative body height separately, females were significantly more slender after 90 days than males, while AI females appeared intermediate (fig. 2B). 
Fig. 2. Comparison of body shape and relative body height. A PCA of overall body shape based on 7 landmarks shows the distribution of the groups along PC 1 and PC 2. Pink circles represent control females, blue circles control males, and purple circles AI females. Filled forms show individuals at day 0 and empty forms after 90 days. Additional outer-dashed lines represent individuals showing (male-specific) territorial coloration. B Relative body height of control females, control males, and AI females shows that groups were highly similar at day 0 [ANOVA: $\mathrm{F}(2)=$ $0.9485, \mathrm{p}=0.4069]$. C Relative body height of control females, control males, and AI females reveals that males developed a deeper body after 90 days that differs from females and AI females [ANOVA, F(2) = 12.842, p < 0.001, Tukey: males/females: $\mathrm{p}<0.001$; males/AI: $\mathrm{p}<0.001$ ]

Fig. 3. Comparisons of head shape and relative head length. A PCA of head shape based on 6 landmarks illustrates the distribution of the groups along PC 1 and PC 2 . Pink circles represent control females, blue circles control males, and purple circles AI females. Filled forms show individuals at day 0 and empty forms after 90 days. Additional outer-dashed lines represent individuals showing (male-specific) territorial coloration. B Relative head length of control females, control males, and AI females shows that groups were highly similar at day 0 [ANOVA, $\mathrm{F}(2)=1.0289, \mathrm{p}=0.3786$ ]. C Relative head length of control females, control males, and AI females reveals that females $(\mathrm{p}<0.001)$ and AI females $(\mathrm{p}<$ 0.001 ) developed a more slender and longer head after 90 days than males, whereas females and AI females did not differ ( $\mathrm{p}=$ $0.161)$.
A

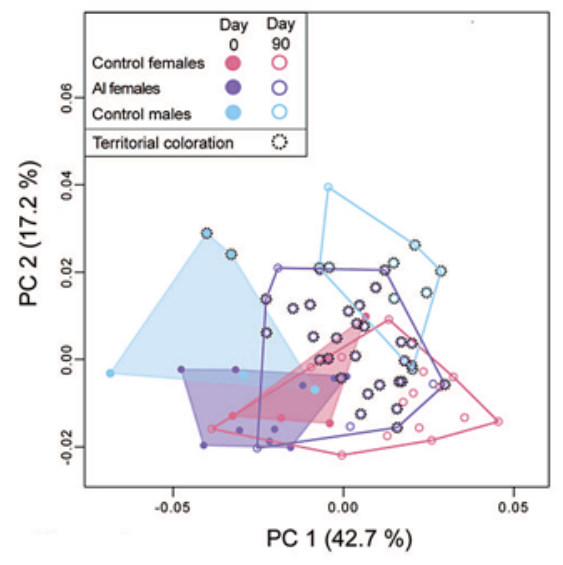

B

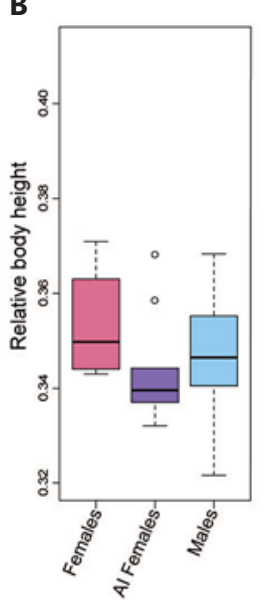

C

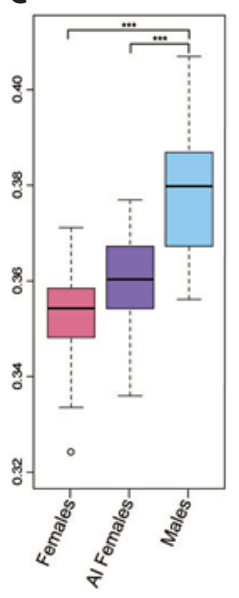

A

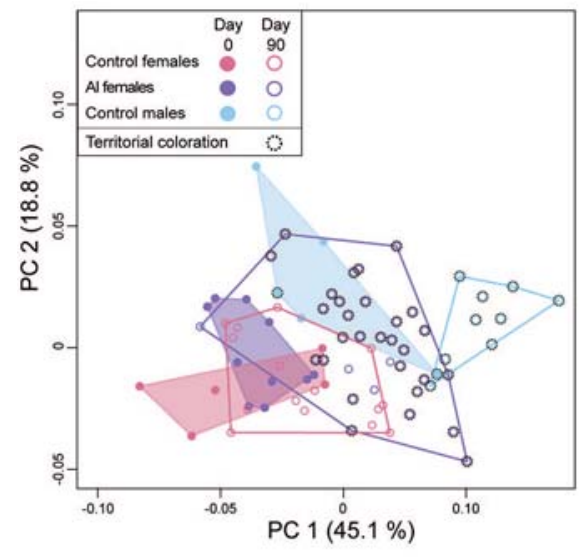

B

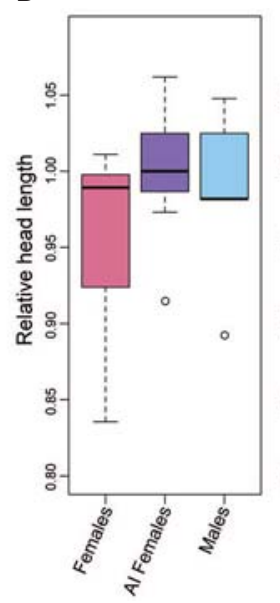

C

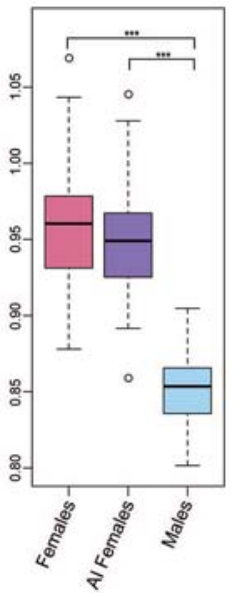

Main variation in head shape (fig. 3A) on PC 1 (accounting for $45.1 \%$ of variance) was based on a shortened head and elongated mouth (with females having a shorter mouth and a more slender but longer head). After 90 days, AI females overlapped with control females on PC 1 and with both males and females on PC 2. After 90 days, head length was significantly different between males and the 2 female groups (fig. 3B).
In conclusion, PCA of head and body shape showed that some AI individuals closely resembled normal females, whereas others developed a shape more similar to males or were placed between a male and female shape.

\section{Hormonal Response to Fadrozole Treatment}

The effect of fadrozole on androgen ( $\mathrm{T}$ and 11-KT) and estrogen (E2) levels was measured during the first 90 days of treatment to assess whether fadrozole indeed in- 
Fig. 4. Hormone levels throughout treatment. 11-KT (A), T (B), and E2 levels (C) for control females and the 2 treatment replicates AI1 and AI2 are shown. For males, hormone baseline levels were assessed only once. Samples were collected before the treatment and after 14, 30, and 90 days of treatment. The levels are shown as means $(\mathrm{ng} / \mathrm{ml}) \pm \mathrm{SEM}$.

Fig. 5. Gonad histology with hematoxylineosin staining. A Female gonad with previtellogenic (PV) and early vitellogenic oocytes (VO). B AI female gonad with previtellogenic oocytes (PV) and a few large oocytes in advanced cortical alveolar stage (O). C Male gonads with testicular tubules and spermatozoids.
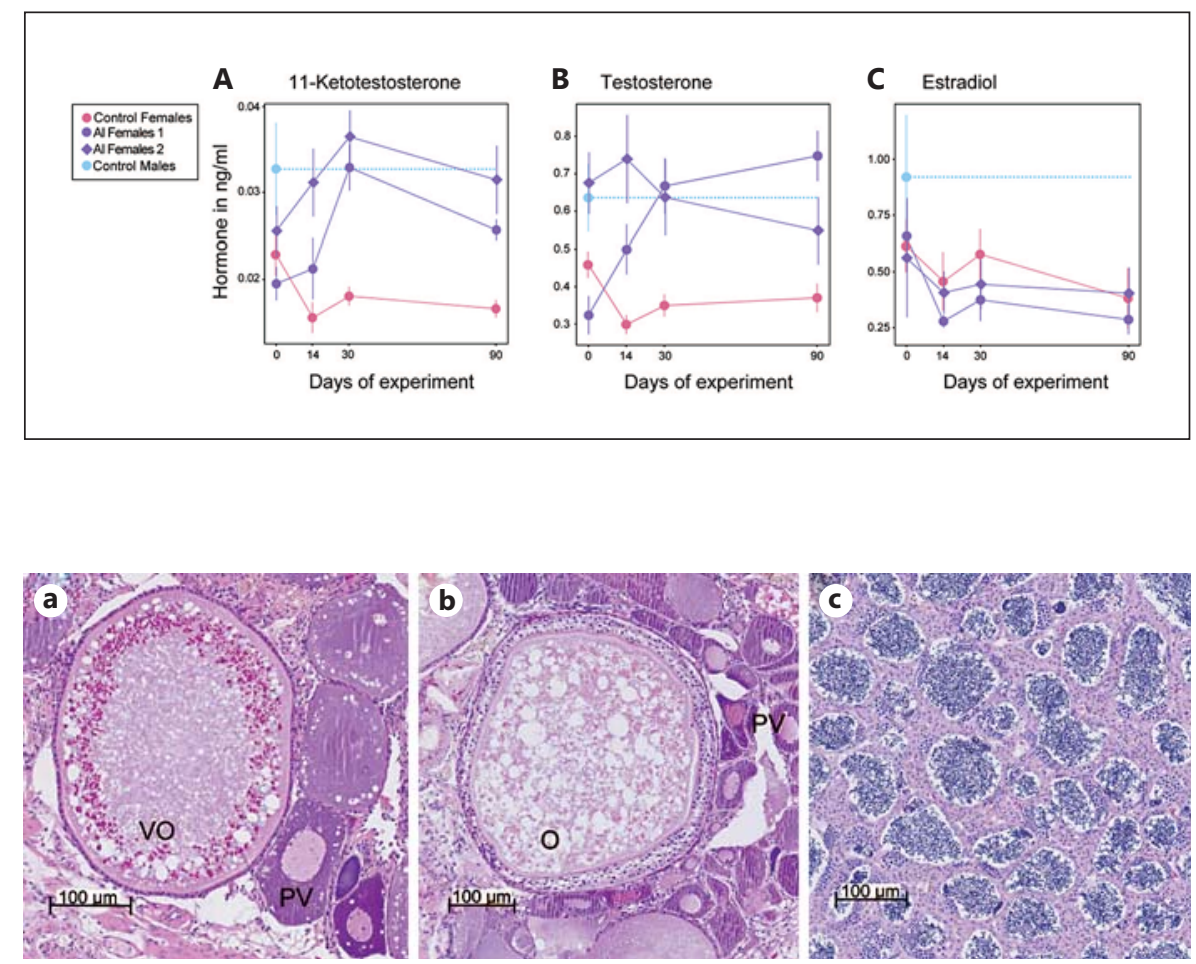

hibits aromatase activity in female $A$. burtoni (fig. 4, same fish as above). T levels differed at day 0 between the $2 \mathrm{AI}$ replicate groups [ANOVA: $\mathrm{F}(3,16)=6.669$, Tukey: $\mathrm{p}<$ $0.01]$ and hence the treatment replicates were analyzed separately.

We observed a significant effect of AI group and treatment day on androgen levels [T: treatment group $\times$ day of treatment: $\mathrm{F}(8,14)=32.276, \mathrm{p}=0.001 ; 11-\mathrm{KT}$ : treatment group $\times$ day of treatment: $\mathrm{F}(8,14)=17.597, \mathrm{p}<$ $0.01]$. During the treatment period, $T$ levels increased continuously in the AI1 females from 0 to 90 days and reached male baseline levels at day 30 with a significant increase already between 0 and 14 days compared to those of control females (fig. 4B; females/AI1: $\mathrm{z}=4.163$, $\mathrm{p}<$ $0.001)$. In contrast, AI 2 fish started with $T$ levels similar to those of control males, and there was no significant effect of day of treatment for this group $[F(3,6)=3.0172$, $\mathrm{p}=0.389$ ]. $\mathrm{T}$ levels of control females decreased between 0 and 14 days $(\mathrm{z}=3.926, \mathrm{p}<0.05)$ and then remained similar until 90 days.

For the second androgen, 11-KT, levels in the 2 treatment replicates were similar at all time points. 11-KT levels increased significantly for both AI groups until day 30 , where they reached male baseline levels (fig. 4A; AI1: significant increase in 11 -KT between 0 and 30 days, $\mathrm{z}=$
3.778, $\mathrm{p}<0.01$ and between 14 and 30 days, $\mathrm{z}=3.488$, $\mathrm{p}=0.00146 ;$ AI2: significant increase between 0 and 30 days, $\mathrm{z}=2.912, \mathrm{p}=0.0108$ ). After treatment started, 11 KT levels of control females were always lower than those of control males and AI females. 11-KT levels of both AI groups differed significantly between 0 and 14 days from those of control females (females/AI1: $\mathrm{z}=2.75, \mathrm{p}<0.01$; females/AI2: $\mathrm{z}=3.243, \mathrm{p}<0.01)$ and remained different until day 90 (females/AI1: $\mathrm{z}=2.972, \mathrm{p}<0.01$; females/ AI2: $\mathrm{z}=2.646, \mathrm{p}<0.05)$.

Estradiol levels were similar in control females and AI females at day 0 and did not significantly change throughout treatment [treatment group $\times$ day of treatment: $\mathrm{F}(7,10)=1.39, \mathrm{p}=0.71$ ] (fig. 4C). As expected, and in agreement with previous studies [O'Connell and Hofmann, 2011; Renn et al., 2012; Huffman et al., 2013; O'Connell et al., 2013], female E2 levels were lower than male levels.

\section{Aromatase Inhibition Does Not Induce Gonadal Sex \\ Reversal in Females}

We analyzed gonad histology after 168 days based on a previous study in Nile tilapia that showed appearance of small spermatogonial cysts after 90 days and fully functional reversal to testis after 180 days of AI treatment 


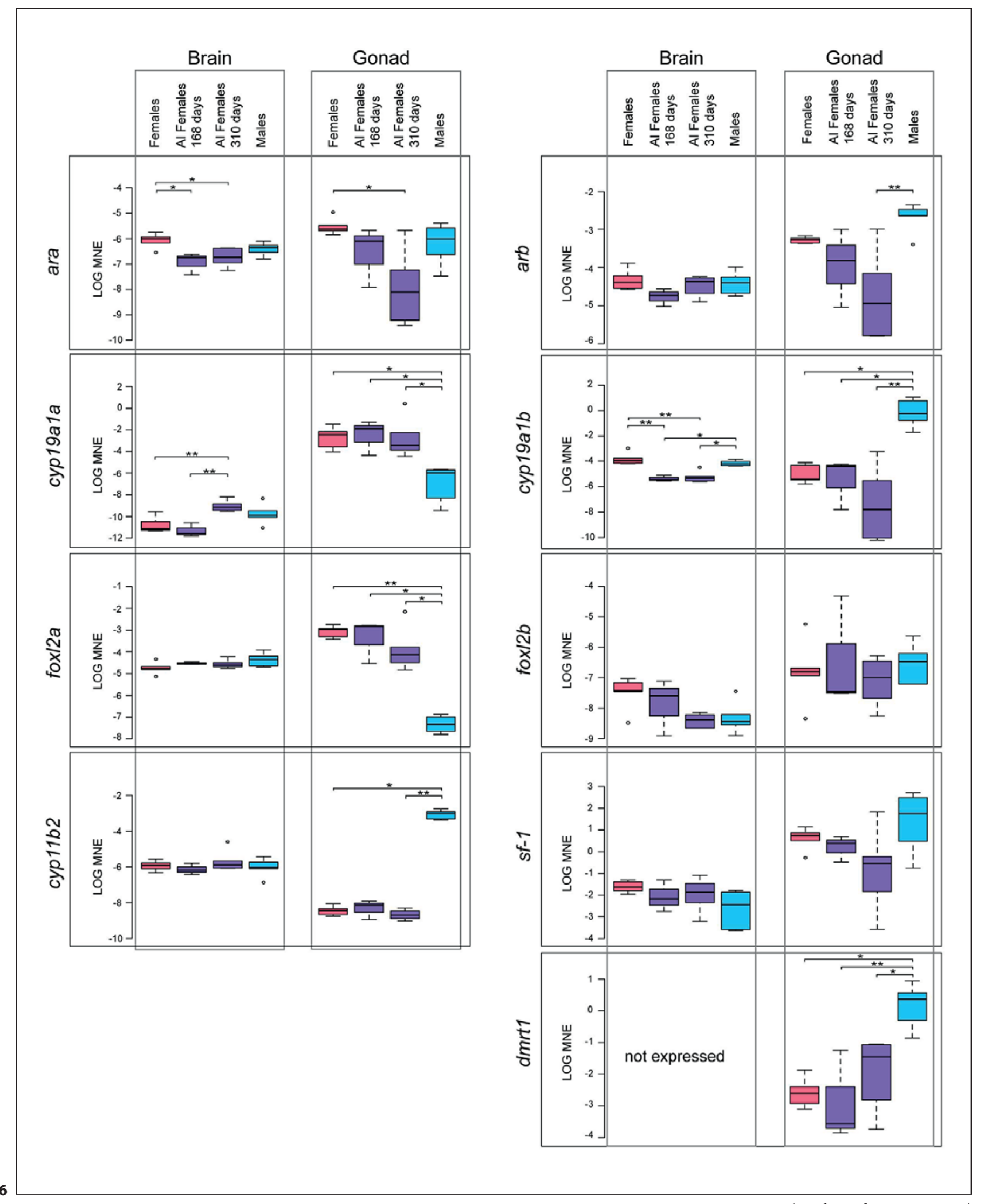

(For legend see next page.) 
[Paul-Prasanth et al., 2013]. Gonad histology of control females showed mature oocytes filled with vitellus, previtellogenic oocytes, and early vitellogenic oocytes as well as atretic large oocytes with vitellus (fig. 5A). Gonads of AI females displayed no similarities in morphology and histology to testis after 168 days with oocytes present in all AI fish analyzed. They showed mainly previtellogenic oocytes (fig. 5B) and a few large oocytes in advanced alveolar stage. Vitellogenesis could not clearly be observed in AI females. Control male gonads showed spermatozoids and cysts of maturing germ cells within testicular tubules (fig. 5C).

The GSI in AI females after 168 days was lower than in female controls and slightly higher than in males (see online suppl. fig. 4 and table 2$)\left(\mathrm{KW}: \chi^{2}=4.461\right.$, d.f. $=3, \mathrm{p}=$ 0.2 ). The GSI increased in all groups, but more in the female controls which had a significantly higher GSI after 310 days than males as well as AI treated females (KW: $\chi^{2}=8.2083$, d.f. $\left.=3, p=0.0165\right)$. Males and AI females showed a similar GSI.

\section{Expression Profiles of Key Genes Involved in Sex Differentiation}

To further interrogate the influence of aromatase inhibition on gonad function, we investigated genetic markers of sexual differentiation and members of the sex steroid pathway in gonads and brain via quantitative realtime PCR. We profiled expression patterns of the aromatases cyp 19a1 a and cyp19a1b, the 2 copies of the androgen receptor $a r a$ and $a r b$, the aldosterone synthase cyp $11 b 2$, the ovarian transcription factors foxl $2 A$ and foxl $2 B$, the potential aromatase regulator $s f-1$, and the conserved vertebrate testis factor $d m r t 1$ again after 168 and 310 days in AI females in comparison to control females and males.

We previously showed that $d m r t 1, c y p 11 b 2$, and cyp19a1b are overexpressed in the testis, whereas cyp19a1 a and foxl2a are overexpressed in the ovaries in A. burtoni [Böhne et al., 2013], and could, therefore, be used as sex-specific markers.

Under the assumption that fadrozole can induce sex change, we expected an upregulation of male-specific

Fig. 6. Expression profiles of key genes involved in sex differentiation in males, females, and AI females. qRT-PCR expression data for ara, arb, cyp19a1a, cyp19a1b, foxl2a, foxl2b, cyp11b2, sf-1, and $d m r t 1$ are shown as mean normalized expression (MNE) [Simon, 2003]. Expression was compared in brain and gonads among females (pink), males (blue), and AI females after 168 and 310 days (purple). Expression of $d m r t 1$ in the brain could not be detected. ${ }^{*} \mathrm{p}<0.05 ;{ }^{* *} \mathrm{p}<0.01$ determined by a Dunn's post hoc test (see online suppl. tables 3, 4 for details).

Adult Sex Change in Astatotilapia burtoni markers and a downregulation of female-specific markers in the gonad of AI females. In the Nile tilapia, a trend in this direction can be observed in AI females [Sun et al., 2014].

In our control fish, we confirmed that the male (cyp19a1b, dmrt1, and cyp11b2) and female (cyp19a1a and foxl2a) gonad markers were expressed in the predicted direction. Furthermore, the previously not tested arb shows a tendency for overexpression in the testis, whereas ara and $s f-1$ did not show a sex specificity in the gonad. Expression patterns are shown in figure 6 (for statistics, see online suppl. tables 3,4 ).

Overall and in contrary to our expectation, AI females at both time points showed gonad expression levels for all genes resembling those of control females. AI females at day 310 showed a significant downregulation in ara and arb. Hence, in agreement with the histological analysis, gene expression in the gonad of AI females remained female-like. Overall, gene expression in the gonad was lower in AI females after 310 days than in AI females after 168 days with the exception of $d m r t 1$.

As a second tissue, we investigated brain expression levels of the same genes. In a previous study, we did not observe significant differences in any of the genes between normal males and females [Böhne et al., 2013]. Here, we observed a significant change in gene expression in the brain of AI females in the genes most directly influenced by the treatment, the aromatase itself (both copies) as well as the androgen receptor a-copy. The aromatase cyp19a1a is significantly upregulated in AI females after 310 days, whereas AI females at 168 days show female-like levels. The second copy, cyp19a1b is significantly downregulated in AI females after 168 and 310 days compared to both control males and females. Compared to control females, also ara is significantly downregulated in AI females after 168 and 310 days, with expression levels closer to males.

\section{Behavioral Response to Fadrozole Treatment}

In a final experiment, we aimed to assess the sexual identity of behavior in the treated fish using a 2-way choice experiment. Focal fish of the 3 groups (females, males, and AI females) were individually presented to a male and female stimulus fish simultaneously, and time spent, interaction time, display behavior, and presence of an eye bar in focal fish were compared among groups.

Time spent with both stimulus fish combined was used as a measure of activity of focal fish, indicating that all groups were equally active (online suppl. fig. 5; for all de- 


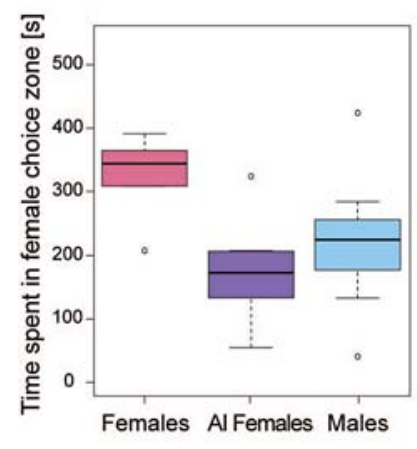

A

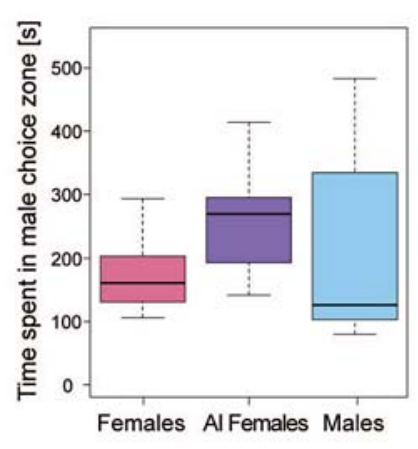

B

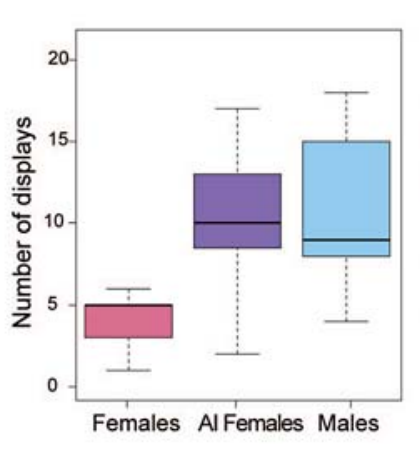

C

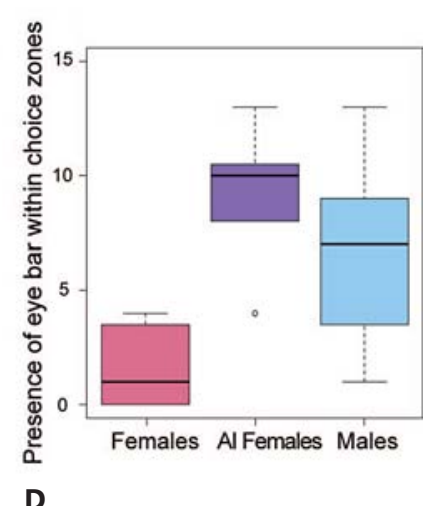

D

Fig. 7. Sex-specific behavior in males, females, and AI females. A Time spent of focal fish in female choice zone. B Time spent of focal fish in male choice zone. C Number of displays shown to both stimulus fish. D Presence of an eye bar within both choice zones.

tails on behavioral experiments, see online suppl. fig. 5-9 and tables 5-8). Overall, AI females showed behaviors that resembled more male-like than female-like behavior (fig. 7).

Focal fish differed in their time spent in the female choice zone $\left(\chi^{2}=6\right.$, d.f. $\left.=2, p=0.04979\right)$, with females spending significantly more time in the female choice zone than AI females ( $p=0.047$ ) (fig. 7A), whereas AI females and males tend to spent more time in the male choice zone than control females (fig. 7A, B).

We also found a significant difference in the number of displays that focal fish showed towards the stimulus fish $\left(\chi^{2}=8\right.$, d.f. $\left.=2, p=0.01832\right)$ (fig. $7 \mathrm{C}$, online suppl. fig. 8 ), with males and AI females showing more displays than females (significantly more in males than females, $\mathrm{p}=0.047$; not different between males and AI females, $\mathrm{p}=1$; AI females and control females, $\mathrm{p}=0.103$ ).

Focal fish are also significantly different in their behavior to show eye bars to focal fish $\left(\chi^{2}=8.963\right.$, d.f. $=2, \mathrm{p}=$ 0.01132 ), with AI females and males showing eye bars more often than females (fig. 7D, online suppl. fig. 9).

\section{Discussion}

Estrogen plays an important role in the regulation of sex differentiation in fishes, with elevated levels inducing ovarian development and maintaining the female phenotype [Baroiller et al., 1999]. Previous studies using AI in females of gonochoristic fishes as distantly related as the Nile tilapia, medaka, and zebrafish revealed that a functional sex change is possible even after sex determination. In the presented study, we tested if the AI fadrozole is also able to induce secondary sex reversal in mature females of the species A. burtoni. In female Nile tilapia, aromatase inhibition causes multiple phenotypic and physiological changes including secondary sex reversal, a reduction in E2 levels, and downregulation of female-specific and activation of male-specific pathway genes [Sun et al., 2014].

We assumed that $A$. burtoni might react similarly to fadrozole treatment as Nile tilapia, since it is also a mouthbrooding cichlid with an XX/XY sex-determining system [Heule et al., 2014b]. Also, aromatase inhibition by fadrozole treatment had previously been shown to work in male $A$. burtoni leading to increased androgen and reduced estrogen levels [Huffman et al., 2013].

Under normal conditions, A. burtoni shows a sex bias in sex steroid hormones, with males having both higher levels of androgens and E2 [O'Connell and Hofmann, 2011; Renn et al., 2012; Huffman et al., 2013; O'Connell et al., 2013]. This is already in contrast to Nile tilapia, where males have lower levels of E2 than females [Sun et al., 2014].

The AI-treated female fish showed a rapid increase in androgen levels, reaching similar concentrations as male fish. However, E2 levels resembled strongly those of control females, with both being actually lower than male levels but no reduction below the female level in AI females. It is possible that a reduction in E2 levels was only tran- 
sient and hence not detected given the time intervals used for our measurements. Note that steroid levels in male A. burtoni treated with fadrozole were measured shortly after injection of the inhibitor [Huffman et al., 2013] and not as in the present study over a period of several weeks. Importantly, in the study by Huffman et al. [2013], T and E2 levels became uncorrelated in male A. burtoni after fadrozole treatment, suggesting a dysregulation of the 2 hormones possibly due to a complex regulatory mechanism. In addition, a compensatory response has previously been shown in other fish species after AI treatment, resulting in a reestablishment of normal E2 levels [Villeneuve et al., 2009a; Garcia-Reyero et al., 2014]. Also, goldfish and common carp treated with fadrozole do not show a reduction in E2 levels [Mikolajczyk et al., 2007]; however, androgen levels were not measured in this study.

We observed a fast reaction to AI in the external phenotype, with treated females showing the appearance of male-typical traits after 14 days already. AI treatment also had some further effects on the overall appearance of the treated fish impacting body and head shape. Previous studies showed that the sexes in cichlids differ mostly in head shape and that mouth-brooding females have larger heads (buccal region) with longer snouts [Oliveira and Almada, 1995; Herler et al., 2010; Van Wassenbergh et al., 2015]. This is in accordance with our findings that females have a more slender but longer head with a shorter mouth, whereas males have a deeper body, a shorter head, as well as longer dorsal and anal fins. Note that prior to treatment, some AI females overlapped with control females but some started closer to males for head and body shape. Some of the AI females developed a body shape that is placed between those of control males and females.

AI females showed a great variation in head shape, with some overlap in morphospace with control females but also occupying a separate part of the morphospace between males and females on PC1. This could indicate that head shape of AI females developed away from female towards male head shape. However, due to sample size differences between time points and groups, it is possible that group variation is underestimated.

None of the females and only a few males displayed territorial coloration at the beginning of the experiment. After 90 days, all control males and $88 \%$ of AI females developed male-typical territorial coloration. Changes in body coloration and egg-spots over time in AI females clearly corresponded to a male phenotype. We propose that the changes in color and shape of AI females are most likely due to the increased levels of androgens and the subsequent activation of androgen specific pathways.

Adult Sex Change in Astatotilapia burtoni
Unlike in the 3 other species studied so far, we did not find male gonadal structures in the gonads of AI females. The GSI was slightly decreased after 168 days in AI females compared to control females and became significantly different after 310 days, being similar to those of males. This suggests that AI negatively affects ovarian maintenance reducing the GSI and possibly impacts oocyte maturation in A. burtoni.

Studies in other fish species showed similar effects on oocyte maturation. Female goldfish treated with fadrozole only contain oocytes at early stages of development [Zhang et al., 2009], and fadrozole reduces circulating plasma vitellogenin concentrations in female fathead minnows [Villeneuve et al., 2009a] and inhibits ovarian growth reflected by a reduction in GSI [Panter et al., 2004]. In zebrafish, fadrozole leads to a disruption of oocyte maturation and ovulation as a consequence of impaired vitellogenesis [Villeneuve et al., 2009b].

With regard to gene expression of male- and femalespecific markers previously found for A. burtoni [Böhne et al., 2013], we could confirm their specificity of expression in our control groups. In AI females, gene expression in the gonad largely resembled the one in control females; especially the aromatase cyp19a1a and cyp19a1b expressions remained at a female-like level. We did not observe the expected shift towards a more male-like pattern. Arb was significantly downregulated in AI females after 310 days compared to control males. In the closest relative, in which gene expression was investigated after AI treatment, the Nile tilapia, gonadal gene expression in AI females changed but global gene expression profiles also remained closer to control females than control males [Sun et al., 2014]. Still, in the Nile tilapia, cyp19a1a increased in the first 45 days of fadrozole treatment followed by a rapid decline below female control levels [Sun et al., 2014], whereas A. burtoni here shows the opposite pattern. In addition, the male-specific markers cyp $11 b 2, s f-1$, and $d m r t 1$ are upregulated in AI females of the Nile tilapia [Sun et al., 2014], a pattern we did not observe.

In fishes, the brain remains active in sex steroid production in the adult stage and is supposedly interacting with the gonad [Le Page et al., 2010]. This becomes evident during sex change in hermaphrodites, which is accompanied by changes in aromatase gene expression in the brain [Breton et al., 2015]. It is hence no surprise that brain gene expression in fishes also changes upon treatment with endocrine disruptors [Tian et al., 2015].

We thus investigated gene expression also in the brain and indeed observed more changes than in the gonads. 
Expression of cyp19a1a in AI females was significantly upregulated after 310 days in the brain compared to control females reaching male expression level, which could account for a compensatory effect in E2 levels. In contrast, cyp19a1b expression was significantly downregulated compared to both control groups, which in addition to the inhibition on the protein level could probably account for the increase in androgen levels. Similar effects on the expression of the brain aromatase cyp19a1b are observed in female medaka [Zhang et al., 2008], goldfish [Zhang et al., 2009], and fathead minnow [Ankley et al., 2002], where cyp $19 a 1 b$ levels significantly decrease in the brain in response to fadrozole treatment. In Melanotaenia fluviatilis fadrozole leads to a decrease in cyp19a1b brain expression in females and to an increase in males [Shanthanagouda et al., 2014]. The situation might be similar in A. burtoni with an overall reduction of cyp19a1b expression in the brain in females treated with fadrozole, whereas males treated with fadrozole show increased aromatase expression in certain brain regions [Huffman et al., 2013].

Furthermore, we detected changes in androgen receptor expression. Two gene copies of the androgen receptor, ara and $a r b$, are present in A. burtoni. Arb usually shows a more widespread distribution in the brain of $A$. burtoni [Harbott et al., 2007]. We detected a downregulation of ara expression in AI females at both time points, resembling more the expression level of males, whereas expression of arb did not change significantly. In A. burtoni, ara is highly expressed in the pituitary, suggesting a role in regulating the hypothalamic-pituitary-gonadal axis [Harbott et al., 2007]. Androgens can regulate the expression of $a r$ in the brain and a downregulation, as the one we observed here in AI females, could be caused by increased testosterone levels [Kumar and Thakur, 2004].

Interestingly, AI females developed some male-typical behavior that differed from female behavior. When given the choice, females tend to spend more time in the female choice zone, whereas males and AI females spent a similar amount of time in the male and female choice zones. This pattern can be explained by the fact that females normally only associate with dominant males at the day of spawning [Kidd et al., 2013]. In addition, males showed significantly more displays than females and in a similar amount as AI females. AI females showed more often an eye bar when visiting choice zones compared to control females, resembling the pattern observed in control males.

In conclusion, the increased levels in androgens coupled with the changes in brain gene expression are likely responsible for the observed phenotypical and behavior- al changes in AI female A. burtoni. However, in contrast to studies in other teleost species, fadrozole did not reduce E2 levels nor induce a full sex reversal and especially did not transform the female to a male gonad in $A$. burtoni, indicating that aromatase and steroids have a different function in sex differentiation and especially in the male and female gonad in A. burtoni than in other species. Effects on coloration and androgen levels were observable rather quickly (after 14 days). This is in agreement with previous studies showing that under normal conditions coloration as well as changes in behavior in combination with changes in sex steroid levels occur rapidly and repeatedly in A. burtoni [Huffman et al., 2013; Carpenter et al., 2014]. This indicates an inherent plasticity of these traits although they are usually coupled to one sex.

Our gene expression data once more underline that the brain and the gonad are both important for sex differentiation and for maintaining sexual identity in fishes. Whereas aromatase levels in the gonad were not impacted, we observed an upregulation of the aromatase a-copy and a downregulation of the b-copy in the brain under AI treatment in females, also suggesting that fadrozole impacts the 2 gene copies differently.

We could previously show that the cyp19a1b coding sequence of $A$. burtoni resembles the one in other fish species, and hence is expected to have a preserved function and thus also susceptibility to fadrozole. However, the a-copy experienced substantial changes at the nucleotide level, likely impacting structure and function of the protein [see fig. 1 in Böhne et al., 2013] and questioning a successful inhibition by fadrozole as well as a conserved role in aromatization of androgens.

Fadrozole coordinates with the iron of the porphyrin nucleus of aromatase, competing with the binding of molecular oxygen to the iron. This reversibly inactivates the aromatase enzyme. Due to its high target specificity it is further assumed that fadrozole specifically interacts with the active site of the enzyme [Browne et al., 1991]. The accession to or composition of different regions of Cyp19a1a might be impacted by the non-synonymous changes that we detected in Lake Tanganyika cichlids.

Our data suggest that the susceptibility to sex reversal is different even between species as closely related as the two cichlids, A. burtoni and O. niloticus. Therefore, impacts of sex steroid hormones and substances interfering with the steroid system need careful investigations on the species and also tissue and physiological level. 


\section{Acknowledgements}

The authors thank Nicolas Boileau for support in the laboratory, Sean Maguire for valuable comments on statistics, and Tessa Solomon-Lane for help with hormone assays. We thank Bernd Egger for advice on behavioral studies as well as him and Marco Colombo for discussions on morphometrics. We also thank Adrian Indermaur for fish keeping and help on photographs. We are grateful to the Waters Corporation (in particular Marijana Petrovic) for providing us with a vacuum manifold. This work was supported by grants from the European Research Council (ERC consolidator grant 'CICHLID X') and the Swiss National Science Foundation (SNF) to Walter Salzburger and the Freiwillige Akademische Gesellschaft Basel (FAG) to Astrid Böhne.

\section{Statement of Ethics}

Research involving animals was performed with approval of the Swiss authorities under the veterinary permits No. 2317 and 2620 issued by the cantonal veterinary office in Basel.

\section{Disclosure Statement}

The authors have no conflicts of interest to declare.

\section{References}

Ankley GT, Kahl MD, Jensen KM, Hornung MW, Korte JJ, et al: Evaluation of the aromatase inhibitor fadrozole in a short-term reproduction assay with the fathead minnow (Pimephales promelas). Toxicol Sci 67:121-130 (2002).

Avise JC, Mank JE: Evolutionary perspectives on hermaphroditism in fishes. Sex Dev 3:152163 (2009).

Baroiller JF, Guiguen Y, Fostier A: Endocrine and environmental aspects of sex differentiation in fish. Cell Mol Life Sci 55:910-931 (1999).

Bates D, Maechler M, Bolker B, Walker S: Fitting linear mixed-effects models using lme4. J Stat Softw 67:1-48 (2015).

-Bhandari RK, Deem SL, Holliday DK, Jandegian $\mathrm{CM}$, Kassotis CD, et al: Effects of the environmental estrogenic contaminants bisphenol A and 17 -ethinyl estradiol on sexual development and adult behaviors in aquatic wildlife species. Gen Comp Endocrinol 214:195-219 (2015).

Böhne A, Heule C, Boileau N, Salzburger W: Expression and sequence evolution of aromatase cyp19a1 and other sexual development genes in East African cichlid fishes. Mol Biol Evol 30:2268-2285 (2013).

Borg B: Androgens in teleost fishes. Comp Biochem Physiol C Toxicol Pharmacol 109:219245 (1994).

- Brawand D, Wagner CE, Li YI, Malinsky M, Keller I, et al: The genomic substrate for adaptive radiation in African cichlid fish. Nature 513:375-381 (2014).

-Breton TS, DiMaggio MA, Sower SA, Berlinsky DL: Brain aromatase (cyp19a1b) and gonadotropin releasing hormone (gnrh2 and gnrh3) expression during reproductive development and sex change in black sea bass (Centropristis striata). Comp Biochem Physiol A Mol Integr Physiol 181:45-53 (2015).

Browne LJ, Gude C, Rodriguez H, Steele RE, Bhatnager A: Fadrozole hydrochloride: a potent, selective, nonsteroidal inhibitor of aromatase for the treatment of estrogen-dependent disease. J Med Chem 34:725-736 (1991).
Carpenter RE, Maruska KP, Becker L, Fernald Heule C, Göppert C, Salzburger W, Böhne A: Ge$\mathrm{RD}$ : Social opportunity rapidly regulates expression of CRF and CRF receptors in the brain during social ascent of a teleost fish, Astatotilapia burtoni. PLoS One 9:e96632 (2014).

Devlin RH, Nagahama Y: Sex determination and sex differentiation in fish: an overview of genetic, physiological, and environmental influences. Aquaculture 208:191-364 (2002).

Forlano PM, Schlinger BA, Bass AH: Brain aromatase: new lessons from non-mammalian model systems. Front Neuroendocrinol 27: 247-274 (2006).

- Garcia-Reyero N, Ekman DR, Habib T, Villeneuve DL, Collette TW, et al: Integrated approach to explore the mechanisms of aromatase inhibition and recovery in fathead minnows (Pimephales promelas). Gen Comp Endocrinol 203:193-202 (2014).

-Green BW, Teichert-Coddington DR: Human food safety and environmental assessment of the use of $17 \alpha$-methyltestosterone to produce male tilapia in the United States. J World Aquac Soc 31:337-357 (2000).

-Harbott LK, Burmeister SS, White RB, Vagell M, Fernald RD: Androgen receptors in a cichlid fish, Astatotilapia burtoni: structure, localization, and expression levels. J Comp Neurol 504:57-73 (2007).

Herler J, Kerschbaumer M, Mitteroecker P, Postl L, Sturmbauer C: Sexual dimorphism and population divergence in the Lake Tanganyika cichlid fish genus Tropheus. Front Zool 7:1-10 (2010).

Heule C, Salzburger W: The ontogenetic development of egg-spots in the haplochromine cichlid fish Astatotilapia burtoni. J Fish Biol 78: 1588-1593 (2011).

Heule C, Salzburger W, Böhne A: Genetics of sexual development - an evolutionary playground for fish. Genetics 196:579-591 (2014a). netics and timing of sex determination in the East African cichlid fish Astatotilapia burtoni. BMC Genet 15:140 (2014b).

-Hofmann HA: Gonadotropin-releasing hormone signaling in behavioral plasticity. Curr Opin Neurobiol 16:343-350 (2006).

Huffman LS, O'Connell LA, Hofmann HA: Aromatase regulates aggression in the African cichlid fish Astatotilapia burtoni. Physiol Behav 112-113:77-83 (2013).

Kidd CE, Kidd MR, Hofmann HA: Measuring multiple hormones from a single water sample using enzyme immunoassays. Gen Comp Endocrinol 165:277-285 (2010).

Kidd MR, O'Connell LA, Kidd CE, Chen CW, Fontenot MR, et al: Female preference for males depends on reproductive physiology in the African cichlid fish Astatotilapia burtoni. Gen Comp Endocrinol 180:56-63 (2013).

Klingenberg CP: MorphoJ: an integrated software package for geometric morphometrics. Mol Ecol Resour 11:353-357 (2011).

- Kumar RC, Thakur MK: Androgen receptor mRNA is inversely regulated by testosterone and estradiol in adult mouse brain. Neurobiol Aging 25:925-933 (2004).

Le Page Y, Diotel N, Vaillant C, Pellegrini E, Anglade I, et al: Aromatase, brain sexualization and plasticity: the fish paradigm. Eur J Neurosci 32:2105-2115 (2010).

Maruska KP: Social regulation of reproduction in male cichlid fishes. Gen Comp Endocrinol 207:2-12 (2014).

Maruska KP, Fernald RD: Reproductive status regulates expression of sex steroid and $\mathrm{GnRH}$ receptors in the olfactory bulb. Behav Brain Res 213:208-217 (2010).

Mikolajczyk T, Sokolowska-Mikolajczyk M, Chyb J, Szczerbik P, Socha M, et al: LH secretion and $17 \beta$-estradiol concentration in the blood plasma and hypothalamus of goldfish (Carassius auratus gibelio B.) and common carp (Cyprinus carpio L.) treated with fadrozole (aromatase inhibitor) and $\mathrm{GnRH}$ analogues. J Anim Sci 52:354-362 (2007). 
Miller WL: Molecular biology of steroid hormone synthesis. Endocr Rev 9:295-318 (1988).

- Nagahama Y: Endocrine regulation of gametogenesis in fish. Int J Dev Biol 38:217-229 (1994).

Nelson JS: Fishes of the World, ed 4, (John Wiley \& Sons, Hoboken 2006).

-O'Connell LA, Hofmann HA: Genes, hormones, and circuits: an integrative approach to study the evolution of social behavior. Front Neuroendocrinol 32:320-335 (2011).

O'Connell LA, Hofmann HA: Social status predicts how sex steroid receptors regulate complex behavior across levels of biological organization. Endocrinology 153:1341-1351 (2012).

O'Connell LA, Ding JH, Hofmann HA: Sex differences and similarities in the neuroendocrine regulation of social behavior in an African cichlid fish. Horm Behav 64:468-476 (2013).

Oliveira RF, Almada VC: Sexual dimorphism and allometry of external morphology in Oreochromis mossambicus. J Fish Biol 46:10551064 (1995).

Panter GH, Hutchinson TH, Hurd KS, Sherren A, Stanley RD, et al: Successful detection of (anti-)androgenic and aromatase inhibitors in pre-spawning adult fathead minnows (Pimephales promelas) using easily measured endpoints of sexual development. Aquat Toxicol 70:11-21 (2004).

- Paul-Prasanth B, Bhandari RK, Kobayashi T, Horiguchi R, Kobayashi Y, et al: Estrogen oversees the maintenance of the female genetic program in terminally differentiated gonochorists. Sci Rep 3:2862 (2013).

- Payne AH, Hales DB: Overview of steroidogenic enzymes in the pathway from cholesterol to active steroid hormones. Endocr Rev 25:947970 (2004).

- Piferrer F, Blázquez M: Aromatase distribution and regulation in fish. Fish Physiol Biochem 31:215-226 (2005).

R Core Team: R: A Language and Environment for Statistical Computing (R Foundation for Statistical Computing, Vienna 2014).

Reed GF, Lynn F, Meade BD: Use of coefficient of variation in assessing variability of quantitative assays. Clin Diagn Lab Immunol 9:12351239 (2002).
Renn SC, Fraser EJ, Aubin-Horth N, Trainor BC, Hofmann HA: Females of an African cichlid fish display male-typical social dominance behavior and elevated androgens in the absence of males. Horm Behav 61:496-503 (2012).

Rohlf FJ: tpsUtil: utility program useful when working with tps files, version 1.60. Department of Ecology and Evolution, State University of New York, Stony Brook (2015a).

Rohlf FJ: tpsdig: digitize landmarks and outlines, version 2.18. Department of Ecology and Evolution, State University of New York, Stony Brook (2015b).

Salzburger W, Mack T, Verheyen E, Meyer A: Out of Tanganyika: genesis, explosive speciation, key-innovations and phylogeography of the haplochromine cichlid fishes. BMC Evol Biol 5:17 (2005).

-Salzburger W, Renn S, Steinke D, Braasch I, Hofmann $\mathrm{H}$, et al: Annotation of expressed sequence tags for the East African cichlid fish Astatotilapia burtoni and evolutionary analyses of cichlid ORFs. BMC Genomics 9:96 (2008).

Santos ME, Braasch I, Boileau N, Meyer BS, Sauteur L, et al: The evolution of cichlid fish eggspots is linked with a cis-regulatory change. Nat Commun 5:5149 (2014).

Schmidt KL, Pradhan DS, Shah AH, Charlier TD, Chin EH, et al: Neurosteroids, immunosteroids, and the Balkanization of endocrinology. Gen Comp Endocrinol 157:266-274 (2008).

Senthilkumaran B: Pesticide- and sex steroid analogue-induced endocrine disruption differentially targets hypothalamo-hypophysealgonadal system during gametogenesis in teleosts - a review. Gen Comp Endocrinol 219: 136-142 (2015).

Shanthanagouda AH, Nugegoda D, Patil JG: Effects of bisphenol A and fadrozole exposures on cyp19a1 expression in the Murray rainbowfish, Melanotaenia fluviatilis. Arch Environ Contam Toxicol 67:270-280 (2014).

Simon P: Q-Gene: processing quantitative realtime RT-PCR data. Bioinformatics 19:14391440 (2003).

Sun LN, Jiang XL, Xie QP, Yuan J, Huang BF, et al: Transdifferentiation of differentiated ovary into functional testis by long term treatment of aromatase inhibitor in Nile tilapia. Endocrinology 155:1476-1488 (2014).
Takatsu K, Miyaoku K, Roy SR, Murono Y, Sago $\mathrm{T}$, et al: Induction of female-to-male sex change in adult zebrafish by aromatase inhibitor treatment. Sci Rep 3:3400 (2013).

Theis A, Salzburger W, Egger B: The function of anal fin egg-spots in the cichlid fish Astatotilapia burtoni. PLoS One 7:e29878 (2012).

- Theis A, Ronco F, Indermaur A, Salzburger W, Egger B: Adaptive divergence between lake and stream populations of an East African cichlid fish. Mol Ecol 23:5304-5322 (2014).

- Tian H, Wu P, Wang W, Ru S: Disruptions in aromatase expression in the brain, reproductive behavior, and secondary sexual characteristics in male guppies (Poecilia reticulata) induced by tributyltin. Aquat Toxicol 162:117125 (2015).

-Van Wassenbergh S, Potes NZ, Adriaens D: Hydrodynamic drag constrains head enlargement for mouthbrooding in cichlids. J R Soc Interface 12:20150461 (2015).

-Villeneuve DL, Mueller ND, Martinovic D, Makynen EA, Kahl MD, et al: Direct effects, compensation, and recovery in female fathead minnows exposed to a model aromatase inhibitor. Environ Health Perspect 117:624631 (2009a).

-Villeneuve L, Wang RL, Bencic DC, Biales AD, Martinovic D, et al: Altered gene expression in the brain and ovaries of zebrafish (Danio rerio) exposed to the aromatase inhibitor fadrozole: microarray analysis and hypothesis generation. Environ Toxicol Chem 28:17671782 (2009b).

Wickham H: ggplot2: Elegant Graphics for Data Analysis (Springer, New York 2009).

Zhang D, Popesku JT, Martyniuk CJ, Xiong H, Duarte-Guterman P, et al: Profiling neuroendocrine gene expression changes following fadrozole-induced estrogen decline in the female goldfish. Physiol Genomics 38:351-361 (2009).

- Zhang X, Hecker M, Park JW, Tompsett AR, Jones PD, et al: Time-dependent transcriptional profiles of genes of the hypothalamicpituitary-gonadal axis in medaka (Oryzias latipes) exposed to fadrozole and 17beta-trenbolone. Environ Toxicol Chem 27:2504-2511 (2008). 